\title{
Filigrane
}

Écoutes psychanalytiques

\section{Violence, terreur infantile et marginalité : sur l'apport de la philosophie de Ricoeur et du récit de soi}

\section{Sophia Koukoui}

Volume 26, numéro 1, 2017

La terreur des enfants : première partie

URI : https://id.erudit.org/iderudit/1041692ar

DOI : https://doi.org/10.7202/1041692ar

Aller au sommaire du numéro

Éditeur(s)

Revue Santé mentale au Québec

ISSN

1192-1412 (imprimé)

1911-4656 (numérique)

Découvrir la revue

Citer cet article

Koukoui, S. (2017). Violence, terreur infantile et marginalité : sur l'apport de la philosophie de Ricoeur et du récit de soi. Filigrane, 26(1), 69-80.

https://doi.org/10.7202/1041692ar
Résumé de l'article

Agités, opposants, turbulents ; les tumultes intérieurs de l'adolescence transcendent parfois les difficultés " classiques » de l'émergence pubertaire pour s'inscrire dans un tout autre registre : celui de la terreur. Ces enfants et ces adolescents " mis à mal " s'adonnent parfois à des agirs transgressifs d'une violence inouïe. Face à l'effraction répétée du système de pare-excitation, aux carences traumatiques primaires et à la violence, comment ces enfants peuvent-ils symboliser ? Comment forgent-ils leur subjectivation ? La violence de ces adolescents nous sidère et nous interpelle tout à la fois. À travers ce texte, l'auteur explore l'articulation entre la littérature, l'écriture de soi et le processus de subjectivation et souligne en quoi une telle démarche d'écriture peut s'avérer thérapeutique et salutaire pour les enfants qui ont connu la terreur. 


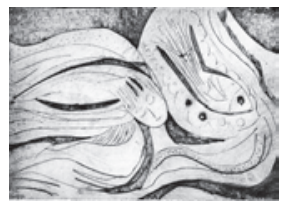

\title{
Violence, terreur infantile et marginalité: sur l'apport de la philosophie de Ricœur et du récit de soi
}

\author{
Sophia Koukoui
}

RÉSUMÉ: Agités, opposants, turbulents; les tumultes intérieurs de l'adolescence transcendent parfois les difficultés «classiques» de l'émergence pubertaire pour s'inscrire dans un tout autre registre: celui de la terreur. Ces enfants et ces adolescents «mis à mal» s'adonnent parfois à des agirs transgressifs d'une violence inouïe. Face à l'effraction répétée du système de pare-excitation, aux carences traumatiques primaires et à la violence, comment ces enfants peuvent-ils symboliser? Comment forgent-ils leur subjectivation? La violence de ces adolescents nous sidère et nous interpelle tout à la fois. À travers ce texte, l'auteur explore l'articulation entre la littérature, l'écriture de soi et le processus de subjectivation et souligne en quoi une telle démarche d'écriture peut s'avérer thérapeutique et salutaire pour les enfants qui ont connu la terreur.

Mots clés: terreur; adolescence; philosophie de Ricœur; narration subjective

\begin{abstract}
Whether they are agitated, oppositional, or unruly, the turbulence and turmoil of adolescents sometimes exceed the typical predicaments faced at puberty. They pertain to another realm: that of terror. These children and adolescents, scorched by life, can act out in exceedingly violent ways. Given the repeated breaks to their protective shield, early maternal deprivation and violence, how can these children access symbol formation? How do they forge their own sense of subjective identity? The degree of violence of these adolescents both astounds and engages us. Throughout this article, the author explores the articulation between literature, self writing and the subjectivation process, and illuminates the ways in which self writing can be therapeutic and salutary for children who have been afflicted by terror.
\end{abstract}

Keywords: terror; adolescence; Ricœur's philosophy; subjective narration.

Quand dans nos services de protection de l'enfance, l'on attribue les agirs des jeunes à une "menace extérieure", le "méchant" en marge de la société [...] c'est que dans cette histoire comme dans tant d'autres problématiques juvéniles, chacun accuse l'autre. Gilbert (2016, argumentaire) 
Toute histoire de souffrance crie vengeance et appelle récit.

Ricœur (1985, p. 115)

\section{Introduction}

a période liminale de l'adolescence n'est pas sans risque d'écueils sur le - plan psychique, l'effraction pubertaire engendrant un retour du refoulé qui fragilise les processus de symbolisation et de subjectivation. Si la période de latence avait permis de poser des représentations stables, l'émergence pulsionnelle adolescente menace de déborder les capacités de liaison du moi. Agités, opposants, turbulents, les tumultes intérieurs de l'adolescence transcendent parfois ces quelques difficultés pour s'inscrire dans un tout autre registre: celui de la terreur. La violence de ceux qui frôlent l'âge adulte nous sidère et nous interpelle tout à la fois. Jusqu'au début du XX $\mathrm{XX}^{\mathrm{e}}$ siècle, l'on imputait à ces adolescents les qualificatifs de "pervers", de "dégénérés»; le champ lexical laissant voir en filigrane la sombre trajectoire qu'on leur destinait. August Aichhorn, un des premiers à introduire la psychanalyse à l'éducation d'adolescents violents, a pour sa part re-conceptualisé l'acte délictueux. Il proposait d'en faire la manifestation d'un trouble affectif suscité par une expérience de frustration ayant sapé l'acceptation du principe de réalité (Aichhorn, 1973; Jeanne, 2005; Queiroz, 2002). Dans son ouvrage Jeunesse à l'abandon, l'éducateur et psychanalyste recommandait de suspendre tout jugement moral, «aussi vain que de prendre parti pour les parents ou pour la société»(Aichhorn, 1973, p. 72). Dans la même lignée, Yves Morhain, qui s'est penché avec acuité sur la question de la violence des adolescents, mentionne que leurs comportements antisociaux «ne se situent pas dans le registre de la transgression de la Loi [...] Ils mettent en scène une disparition subjective et/ou une lutte désespérée pour l'existence» (Morhain, 2009, p. 105). Les angoisses archaïques d'anéantissement, d'abandon et d'intrusion que ces adolescents ne parviennent pas à élaborer sont momentanément évacuées dans la désubjectivation de l'autre. En effet, les enjeux se situent bien en deçà de la transgression de la Loi et le parti pris semble futile.

L'incipit de mon introduction soulignait la défaillance de symbolisation et de subjectivation chez les adolescents; enjeux particulièrement sensibles pour ceux d'entre eux qui ont connu la terreur ou les traumatismes primaires. La thèse que je soutiendrai dans cet article est que l'élaboration et le traitement des traumatismes psychiques peuvent être vectorisés par la dialectique entre lecture et écriture de soi. Les réflexions fécondes de Ricœur 
sur l'identité narrative et le récit de soi m’apparaissent des plus propices à cette analyse. Je propose donc de jeter quelques bases conceptuelles psychanalytiques, pour ensuite aborder le récit de soi au prisme de la philosophie ricœurienne. Loin d'être exhaustive, cette incursion dans l'œuvre du philosophe vise à présenter quelques concepts princeps, qui serviront de matrice à cet article. Dans un troisième temps, la vignette clinique de Diego, un adolescent institutionnalisé, servira d'illustration à ce que le récit de soi recèle de potentialités thérapeutiques face à la terreur. Car si l'on aborde peu les récits de soi des enfants, ceux des enfants institutionnalisés qui ont connu leur lot de violences font essentiellement défaut aux champs psychanalytique et littéraire. Cet article vise à mettre en lumière ce que leur processus de narration subjective révèle de la pugnacité de leur survivance et comment il peut constituer une avenue thérapeutique. Il est donc question d'écrits qui se veulent libérateurs et subjectivants, particulièrement pour ces êtres qui ont été dépossédés d'eux-mêmes par de stériles souffrances et pour lesquels le récit narratif constitue a minima une avenue de reconstruction de soi, voire la voie royale.

\section{De quelques considérations psychanalytiques et littéraires}

Avant de parcourir certains concepts névralgiques de la philosophie ricœurienne, je propose dans la présente section quelques postulats psychanalytiques et littéraires.

Premier postulat: le récit est organisateur de la pensée, parce que le langage structure la pensée.

«Les mots sont magiques dans leur capacité d'influer l'esprit de ceux qui s'en servent. "Ce ne sont que les mots", nous disons avec mépris, oubliant que les mots ont le pouvoir de façonner la pensée des hommes, de canaliser leurs émotions, de diriger leurs volontés et leurs actes » (Kelley-Lainé, 2014, p. 83). Cette citation de Huxley, reprise en français par Kelley-Lainé, fait part de la manière dont le langage façonne la pensée, la structure - idée qui n'est pas étrangère aux écrits de Lacan, Lévi-Strauss, Foucault et Spivak, pour ne citer que ces derniers. Or, si le langage revêt cette fonction, la mise en mots de l'histoire du sujet à travers le récit narratif structure tant sa pensée que son intériorité psychique.

Deuxième postulat: le récit narratif permet de donner sens à l'histoire du sujet et de la rendre intelligible.

Le sens est ici considéré dans son acception polysémique. À travers les dédales de la mémoire, les affres de l'existence, les regards infiniment 
changeants que le sujet pose sur lui-même, le sens de l'histoire d'une vie est en perpétuelle transformation. L'entreprise d'un récit d'une vie me semble une invite intime, de soi à soi, à faire sens des événements et rendre intelligible leurs réverbérations dans la psyché - s'écrire pour se comprendre, dans un processus de liaison/déliaison qui est le propre des processus épistolaire et intrapsychique. De plus, face à une expérience de terreur, l'écriture de soi peut soutenir le développement de la fonction alpha (Bion, 1962).

Troisième postulat: le récit se crée dans la relation à l'Autre.

L'Autre est l'indispensable interlocuteur à travers lequel le récit va pouvoir se déployer. Il n'existe pas de récit qui soit de soi à soi. L'affirmation de Ricœur selon lequel le plus court chemin de soi à soi passe par autrui (Ricœur, 1990) trouve écho dans la définition de l'être humain de Nasio, selon lequel « tout être humain quel qu'il soit et quelle que soit sa souffrance, veut parler à un autre. Si nous devions définir l'être humain nous dirions: un humain est celui qui a la volonté irréductible, le besoin impérieux de communiquer avec un autre humain. Voilà le principe souverain, la prémisse indiscutée qui préside à toute écoute analytique» (Nasio, 2007, 28). Ainsi, le lecteur devient un témoin des plus précieux, tel qu'illustré brillamment par Chiantaretto, qui ouvre sur la fonction conative de l'écriture, soulignant la manière dont le lecteur est interpellé comme «témoin garant d'une écoute» (Chiantaretto, 2014).

\section{Identité et récit de soi : quelques notions princeps de Paul Ricœur}

La notion d'identité se décline en plusieurs instances dans l'œuvre de Ricœur. Son analyse se déploie initialement dans les trois tomes de Temps et récit, où il est question d'une philosophie herméneutique du temps, pour se poursuivre dans Soi-même comme un autre, où il développe entre autres la notion d'identité narrative. Les concepts de temporalité et d'identité sont imbriqués dans la trame de son œuvre et procèdent d'une tension où ces deux concepts s'articulent, se font écho et s'enchevêtrent tout à la fois. Notamment, Ricœur se questionne sur la permanence du sujet à travers le temps et la multiplicité de ses expériences. Face à l'aporie du temps et de l'unicité de soi ${ }^{1}$, il sonde les bases d'une continuité de soi-même et soutient que ce qui permet de rassembler la multiplicité de nos expériences n'est guère une quelconque substantialité sous-jacente comme le théorisaient les philosophes idéalistes, mais plutôt l'intelligibilité que le sujet donne à son récit de vie. Ricœur aborde trois modalités de l'identité: l'identité-idem, qui renvoie 
à la notion de caractère; l'identité-ipse, qui constitue un défi au temps et un déni de changement - «quand même mon désir changerait, quand même je changerais d'opinion, d'inclinaison, “je maintiendrais"» (Ricœur, 1990, p. 149); puis l'identité narrative que j'élaborerai davantage au fil du présent texte ${ }^{2}$. Elle est, selon Ricœur, la seule qui puisse «inclure le changement, la mutabilité, dans la cohésion d'une vie» (Ricœur, 1985, p. 443) et elle correspond à la capacité à mettre en récit les événements de l'existence du sujet. Or, selon Ricœur, cela n'est faisable que par la fréquentation de récits historiques ou de fiction. Le philosophe met en exergue l'importance de l'appropriation critique, où le lecteur trouve une résonance particulière à son vécu subjectif dans l'histoire du personnage. Ainsi, un transfert s'opère de l'identité du personnage à l'identité du sujet.

Nous ne pouvons aborder le concept d'identité narrative en passant sous silence l'étayage de Ricœur sur la notion d'intrigue dans la Poétique d'Aristote. Pour ces deux philosophes, le récit consiste à agencer des faits et des événements pour en faire une histoire. Le récit se trame au fils de concordances et de discordances: les premières rendant l'histoire intelligible, les deuxièmes correspondant à des renversements qui viennent troubler le cours du récit mais qui permettent en définitive de faire progresser l'histoire. L'intrigue survient lors d'une scission, d'un épisode discordant, inattendu ou invraisemblable. Le travail de mise en récit consiste donc à inscrire cet événement (possiblement traumatique) dans la trame narrative, de manière à ce que l'invraisemblable devienne concordant avec ce qui l'a précédé. Cette transformation, qu'Aristote appelait muthos, Ricœur la nomme configuration, cette dernière consistant en «cet art de la composition qui fait médiation entre concordance et discordance» (Ricœur, 1990, p. 168). En somme, l'événement «discordant» devient indispensable à l'histoire du sujet: un événement sans lequel il eût été un Autre. À travers la lecture historique ou de fiction s'opère un transfert de l'identité du personnage du récit à l'identité du lecteur, processus que Ricœur a nommé la refiguration. Le récit est donc une "proposition de monde» qui appelle à un retour à la vie ellemême, pour transformer son identité propre. Ainsi, le récit de soi ne cesse d'être repensé par l'ensemble des histoires que le sujet se raconte sur luimême, laissant libre cours aux interrogations, aux assertions, aux paradoxes.

Il en va de même au sein de l'expérience analytique, alors que le sens n'est jamais circonscrit ni définitif, mais plutôt en constante élaboration; alors que le sujet revisite son histoire et fait des incursions itératives au cœur de lui-même. Ainsi, plutôt qu'une identité sclérosée par le temps, le récit 
permet une fluidité et une évolution de l'identité du sujet. Tissu d'histoire et tissu psychique procèdent donc d'une textualité toujours renouvelée (Fortin, 2007).

Les pôles d'articulation entre la philosophie ricourienne et la psychanalyse sont multiples. Cette introduction visait à présenter certaines bases conceptuelles afin d'asseoir, sur le plan théorique, l'apport de l'écriture de soi à la psychanalyse. Afin d'illustrer mon propos, la section qui suit aborde le cas d'un jeune adolescent caractérisé comme «violent».

\section{Le cas de Diego}

Diego est un adolescent d'Amérique Centrale, dont la famille s'était établie au Canada alors qu'il était en bas âge. Issue d'un milieu fort modeste, la famille habitait un logement précaire et s'alimenter constituait un enjeu. Diego a connu des carences tant nutritives qu'affectives. Outre les difficultés matérielles, sa mère éthylique était psychiatrisée, ce qui a fait en sorte que Diego a peu été bercé à la douceur de la portance psychique maternelle. Il avait également connu plusieurs pertes successives de figures paternelles dans les premières années de vie. C'est dans un contexte de négligence et d'une incapacité parentale à assurer tant sa protection que son développement que Diego a été placé en institution à l'âge de 7 ans. De la période de latence à sa jeune adolescence, il voyait sa mère sporadiquement à l'extérieur du foyer - des retrouvailles toujours trop courtes. Puis, la mère de Diego est décédée d'une insuffisance cardiaque, quelques mois avant que je ne le reçoive en consultation.

La demande d'évaluation qui m'a été adressée était instiguée par le milieu institutionnel où résidait Diego. La requête faisait part d'une hypothèse diagnostique de prodrome schizophrénique. Diego avait un mode de vie nocturne et il s'isolait passablement. Il avait tissé peu de liens d'amitié avec les jeunes du centre et, dans la dernière année, ses contacts avec les autres adolescents avaient été ténus. Le personnel soignant s'inquiétait de ses propos sur la fin du monde. De plus, il avait eu des démêlés avec la justice en raison d'actes délictueux: menace de mort, vols à l'étalage et transactions frauduleuses sur internet, toujours dans le but de se constituer un trousseau de survie en préparation pour l'apocalypse. Ces indices, adjoints à l'historique familial de fragilité psychique, ont fait appréhender au personnel soignant un glissement vers la psychose.

En me dirigeant vers la salle d'attente pour accueillir Diego, j'ai été happée par son regard, empreint d'une peine abyssale. Ses yeux criaient la 
tristesse. Toutefois, l'adolescent a rapidement fait montre d'aisance dans mon bureau, comme on le voit souvent chez les enfants institutionnalisés pour lesquels les rencontres avec le personnel soignant sont récurrentes. Il m'a fait part d'événements traumatiques, entre autres d'une tentative homicidaire de sa mère sous ses yeux, une tentative d'enlèvement par un inconnu près du domicile familial et des séparations répétées de sa mère sans qu’il sache quand il la reverrait. Tout cela était relaté presque sans affect. Je l'ai questionné sur son parcours, sur ce qui l'intéressait, sur la vie au centre. Il m'a fait part d'une vacuité dans un foyer dont il récusait sans ambages les modes de fonctionnement et auquel il imputait ses difficultés actuelles. Le «système» relevait ses actes délictueux et, en miroir, il blâmait l'institution de faillir dans son rôle éducatif. Tout lui semblait futile, dérisoire. La nuit, alors que les autres dormaient, il écrivait des nouvelles dans sa chambre... à la première personne du singulier. Il me fit part de son intérêt pour la littérature et de son projet de devenir écrivain.

Au cours de ce premier entretien, nous avons ouvert sur les vols que Diego avait commis. Ces derniers étaient accompagnés d'une exaltation narcissique et il voyait ses actes délictueux comme une revanche face aux riches qui «ont tout». Il refusait d'être inféodé à l'hégémonie sociale; système inique où les plus nantis connaitraient un sort plus favorable au moment de l'apocalypse. À travers ces agirs transgressifs, il se préparait à la fin du monde qui, disait-il, était imminente. Un jour, la planète serait surpeuplée et il n'y aurait plus suffisamment de ressources pour tous. Seuls les riches survivraient. Il lui fallait donc se munir de réserves.

En ce qui a trait à la figure maternelle, Diego faisait une distinction sans équivoque entre les privations matérielles de sa prime enfance et l'environnement affectif dans lequel il avait baigné. Son discours manifestait une idéalisation flagrante de l'imago maternel, nécessaire et protectrice à la lumière de son histoire. Je lui demandai si son intérêt pour l'au-delà n'était pas issu d'un questionnement visant à savoir où était sa mère depuis son décès, ce à quoi il me répondit: "Tengo miedo que se quede en las sombras. Quiero enterarme de la vida después de la muerte porque quiero saber dónde està ${ }^{3}$.» C'était donc cela, sa fascination pour le monde occulte. Elle émanait d'une inquiétude pour l'âme de sa mère, ce qu'elle était advenue, où elle était. Il me confia alors qu'il essayait de trouver des preuves de l'existence de Dieu.

De ce premier entretien avec Diego était ressorti une inscription ténue dans le lien social et une affliction morale chez cet adolescent qui amorçait à 
peine le deuil de sa mère. Le décès de cette dernière était le point focal de sa scène interne, dont les agirs étaient une manifestation indéniable. Avant de partir, je lui demandai de venir à la prochaine rencontre avec un objet qui le représente bien.

Diego s'est présenté à la séance subséquente, objet transitionnel en main. Il avait apporté son recueil de nouvelles. Je lui ai demandé d'en sélectionner une et de bien vouloir me la lire. Dès la première phrase, il racontait le départ de la figure maternelle et son esseulement dans un lieu isolé, où se trouvait une machine pour se divertir. Dans son histoire, il éprouvait au départ de sa mère une expérience sombre et angoissante, dans un environnement toxique et potentiellement mortifère. Il pleurait doucement, puis paniquait et hurlait. Les vécus hallucinatoires étaient couplés d'angoisses d'anéantissement et d'agirs sadiques. Le récit narratif de Diego faisait écho à ses expériences de terreur et de carences primaires. L'adolescent me permit de photocopier son histoire et je l'assurai que si j'en faisais mention dans le rapport psychologique, je ne traiterais que de bribes d'histoire afin de respecter les droits d'auteur.

Lui demandant quel était son auteur préféré, il me répondit Adolfo Bioy Casares. Nulle surprise quant à l'attrait de Diego envers l'œuvre de l'écrivain, qui plonge le lecteur dans un univers sombre et patibulaire, où les protagonistes se butent à des puissances autoritaires. Les écrits de Bioy Casares témoignent d'un désir de se soustraire à la linéarité du temps, qui conduit l'Homme au sabbat des sabbats. Le thème de son œuvre maîtresse, L'invention de Morel (Bioy Casares, 1992), résonne considérablement avec les préoccupations et le vécu de Diego. Ce classique de la littérature contemporaine est une douloureuse métaphore sur la solitude, dans laquelle le personnage principal, psychiquement fragile, se retrouve esseulé sur une île déserte après avoir eu des démêlés avec l'appareil judiciaire. Le héros, ou anti-héros, est invisible aux yeux de ceux qui l'entourent. Il réglera finalement la machine de Morel de manière à maintenir à l'infini le lien à son objet d'amour.

Sentiment d'invisibilité, esseulement, agirs violents et transgressifs, les investissements littéraires de Diego faisaient écho à sa propre histoire et ses processus identificatoires furent mis en branle par le récit d'un héros dont le vécu résonnait avec le sien. C'est en ce sens que sa lecture de L'invention de Morel permit d'opérer une véritable appropriation critique, au sens ricœurien du terme. Diego trouva écho à ses expériences dans la mise en intrigue de L'invention de Morel. Il sut ensuite revenir à sa propre histoire par un processus de refiguration, approfondir ses pensées par son processus d'écriture de soi et 
commencer à subjectiver les événements de sa vie. Aussi pouvons-nous dire que l'auteur, Bioy Casares, a tenu un rôle d'instance tutélaire in absentia, dont la présence métonymique par l'objet du roman nourrissait les pensées de cet adolescent et lui offrait une présence. Au-delà d'un mécanisme de sublimation, le récit de soi de Diego sous-tendait son processus de subjectivation et la consolidation de son identité. Son engagement épistolaire constituait un processus d'élaboration secondaire, particulièrement face à une expérience de terreur pour cet adolescent qui s'écrivait la nuit dans l'ombre, alors que les autres dormaient. Enfant endeuillé qu'il était, cette écriture de soi avait œuvré à la pérennisation d'un moi enchevêtré dans des pulsions mortifères. Le traumatisme de la perte de l'objet primaire, qui peut mettre en danger la capacité de métaphorisation, avait été préservé, voire cultivé, par son écriture de soi. En accédant à sa propre histoire, il accédait à lui-même.

\section{La restitution}

Au niveau étymologique, le terme latin restitutio évoque à la fois reconstruction et restauration. Il s'agit de rendre quelque chose que l'on possède indûment, de rétablir. En agriculture, la restitution correspond au retour au sol des résidus de récolte; acception qui évoque le retour aux racines. En étendant la métaphore agricole au champ clinique, la restitution consiste à semer chez le patient quelque chose de lui-même qui va pouvoir germer et mûrir en lui. Au-delà du psycho-diagnostic, il s'agit d'un des premiers actes thérapeutiques. Pour tout patient et peut-être davantage pour les êtres marginalisés, un diagnostic peut relever du commentaire politique et social et peut restituer à l'Autre sa part d'humanité (Fernando et Ferns, 2010; Moodley et Ocampo, 2014).

Ainsi, pour Diego, mon rapport faisait état de l'histoire de terreur d'un adolescent qui avait été écorché vif et dont les traumatismes infantiles se téléscopaient à la période mutative de l'adolescence. Ses agirs transgressifs étaient les manifestations d'une détresse profonde inscrite dans une trajectoire marquée par la terreur et la "violence par le manque» (Richard, 1997). Ma compréhension de sa destructivité était davantage de l'ordre de la tendance antisociale de Winnicott (Winnicott, 1956). En effet, les larcins commis pour se constituer un kit de survie pouvaient être appréhendés à plusieurs niveaux. Outre leur adhérence au scénario d'une catastrophe malthusienne, ils s'inscrivaient dans l'inconscient collectif d'images apocalyptiques et témoignaient également de son expérience de déprivation. Pour reprendre l'expression de Lebovici, Mâle et Pasche, ces vols étaient 
des «compensateurs de frustrations affectives» (Lebovoci et al., 1951). La période où les vols avaient été commis revêtait une importance capitale. En effet, Diego n'avait commencé à voler que suite au décès de sa mère. Or, comme le souligne Dominique Caïtucoli, «qu'il prenne de l'argent à sa mère, à un proche ou, de façon plus anonyme, à la société, derrière le passage à l'acte il y a pour l'enfant ou l'adolescent le fantasme de la création primaire, celui de créer le sein là où il l'attend et où la mère le lui présente» (Caïtucoli, 2005). En d'autres termes, le vol est aussi un appel à la mère archaïque, retrouvée le temps du passage à l'acte.

Quant aux préoccupations de Diego pour la fin du monde, elles s'inscrivaient également dans le sillage d'un traumatisme primaire. Je mentionnais dans le rapport que la fin du monde était déjà survenue pour lui. Il s'agissait d'un enfant qui avait perdu sa mère deux fois: en étant institutionnalisé et lors de son décès. Or, y a-t-il événement plus apocalyptique pour un enfant que le décès de sa mère? Ainsi, sans occulter la violence de Diego, il s'agissait d'ouvrir la voie sur d'autres interprétations, dans une conceptualisation du psycho-diagnostic qui se voulait polysémique et ouverte tant sur la scène interne que sur la réalité du monde.

\section{Conclusion}

J'ai toujours été fascinée par l'intuition des enfants, qui orientent leur choix d'objet vers ce qui - et je le dirais de façon tout à fait triviale - est «bon pour eux». Diego a certes une histoire singulière et l'écriture lui a été salutaire. D'autres enfants ont également recours à l'écriture de soi, sous la forme de nouvelles ou de poèmes. Ils nous en apportent souvent des fragments en séance, ou les composent à même l'espace thérapeutique. J'aurais pu écrire sur la narration de soi d'un autre enfant qui a connu la terreur. Mon choix a été animé par mes motions contre-transférentielles dans l'après-coup, puisque c'est plusieurs mois après la remise de mon rapport à l'institution que, dans une forme inchoative, le lacis des premières idées de ce manuscrit a émergé. Je n'ai jamais revu Diego pour une rencontre de restitution et son histoire a continué de m'habiter. Écrire son histoire correspondait indubitablement à un fantasme de continuer d'être présente pour lui et d'exercer une fonction contenante pour son histoire. Peut-être devrais-je dire, une matrice à son histoire, puisque le transfert maternel allait de soi.

Comme le soutient Ricœur dans le troisième tome de Temps et Récit, «le soi de la connaissance de soi est le fruit d'une vie examinée, selon les mots de Socrate dans l'Apologie. Or, une vie examinée est, pour une large part, une 
vie épurée, clarifiée par les effets cathartiques des récits tant historiques que fictifs véhiculés par notre culture. L'ipséité est ainsi celle d'un soi instruit par les œuvres de la culture qu'il s'est appliquées à lui-même [...]. Un sujet se reconnaît [donc] dans l'histoire qu'il se raconte à lui-même sur lui-même» (Ricour, 1985, p. 365). Et quant à Diego, cet enfant mal accueilli, au sens ferenczien du terme (Ferenczi, 1929), s'écrire relevait d'une autopoesis: se donner naissance à travers l'écriture de soi.

\author{
Sophia Koukoui \\ sophia.koukoui@mail.mcgill.ca
}

\title{
Notes
}

1. En d'autres termes, Ricoeur problématise la question de la permanence du sujet à travers le temps et ses expériences de vie.

2. Pour une analyse plus exhaustive de l'identité narrative dans l'œuvre de Ricoeur, le lecteur peut se référer à "Narrativité, narration, narratologie: du concept ricœurien d'identité narrative aux sciences sociales» de Johann Michel, paru dans la Revue européenne des sciences sociales, mis en ligne le $1^{\text {er }}$ décembre 2009. URL: http://ress.revues. org/562.

3. «J'ai peur qu'elle soit dans l'obscurité. Je veux savoir ce qu'il y a après la mort, parce que je veux savoir où elle est» (traduction libre).

\section{Références}

Aichhorn, A. (1973). Jeunesse à l'abandon. Toulouse: Privat.

Aristote. (1980). La Poétique, Paris: Seuil.

Bion, W., (1962). Aux sources de l'expérience, Paris: Presses universitaires de France.

Bioy Casares, A. (1992) L'invention de Morel. Paris: édition domaine étranger 10/18.

Caïtucoli, D. (2005). Winnicott : volet, détruire, l'appel au secours ou la tendance anti-sociale. Filigrane. 14 (1). 33-54.

Chiantaretto, J-F., (2014). «S'écrire: survivre ou se faire naître dans le regard de l'autre? A propos de l'écriture de soi chez Imre Kertész». Dans Eva Brabant-Gero (dir.). S'écrire (Le Coq-Héron 219, p. 46-49) Paris : Érès.

Ferenczi, S., (1929) L'enfant mal accueilli et sa petite mort, Psychanalyse, Oeuvres Complètes, tome V, traduction en français, Paris: Payot, 1968-1982.

Fernando, S. \& Ferns, P. (2010) The bigger picture. Mental Health Today, p. 25.

Fortin, B., (2007). Freud: une fondation apoétique de la psychanalyse?. Dans Eva Brabant (dir.) L'expérience analytique en tant qu'expérience poétique (Le coq-héron, vol. 189 p. 19-27), Paris: Érès.

Jeanne, Y. (2005). August Aichhorn. Prendre en compte la «jeunesse à l'abandon». Reliance, 18 (Liberté et responsabilité dans la vie affective, familiale et sexuelle), 132-139.

Kelley-Lainé, K. (2014). L'écriture de soi... s'écrire l'intime. Le coq-héron, 219 (S'écrire), 83-88.

Lebovici, S., Mâle, P., et Pasche, F. (1951). Psychanalyse et criminologie. Rapport clinique au $13^{\text {e }}$ Congrès des psychanalystes de langue française. Revue française de psychanalyse, 15 (1), 30-61. 
Moodley, R., et Ocampo, M. (2014). Critical Psychiatry and Mental Health: Exploring the work of Suman Fernanco in clinical practice. New York: Routledge Taylor and Francis Group.

Morhain, Y. (2009). Adolescents criminels: une haine envieuse. Dans Y. Mohrain et R. Roussillon (dir.). Actualités psychopathologiques de l'adolescence (p. 73-108) Louvainla-Neuve: De Boeck.

Nasio, J.-D. (2007). Mon corps et ses images. Paris: Payot.

Queiroz, P. (2007). Aichhorn, un autre regard sur la délinquance juvénile. Dans Enfances et Psy, 35 (2), 144-146.

Richard, H. (1997). Dis-moi qui tuer? Violence dans le social et en situation thérapeutique. Présentation. Filigrane, 6(1), 3-4.

Ricœur, P. (1983). Temps et Récit, Tome I, Paris: Seuil.

Ricœur, P. (1984). Temps et Récit, Tome II, Paris: Seuil.

Ricœur, P. (1985) Temps et Récit, Tome III, Paris: Seuil.

Ricœur, P. (1990) Soi-même comme un autre. Paris: Seuil.

Winnicott, D. W. (1976). La tendance antisociale. Dans De la pédiatrie à la psychanalyse. Paris: Payot, 1956. 